\section{Crossing over to bispecificity}

\section{By Chris Cain, Staff Writer}

Researchers at Roche have developed a way to produce bispecific antibodies from existing mAbs. ${ }^{1}$ By preserving structural similarities to the minimally immunogenic IgG backbone used by most $\mathrm{mAb}$ therapeutics, the approach could minimize immunogenicity and provide pharmacokinetics on par with those of natural antibodies while granting the ability to inhibit two targets with a single molecule.

Previous approaches for developing therapeutic antibodies that bind more than one antigen typically involved the complex engineering of multiple antigen-binding domains into a single molecule. ${ }^{2}$ These approaches often created new, non-natural antibody structures with the potential for increased immunogenicity or altered pharmacokinetics that could lead to rapid clearance from the bloodstream. In addition, the antibodies were difficult to manufacture.

To avoid these complications, a team at Roche's Pharma Research and Early Development (pRED) unit sought to create a bispecific antibody that closely resembled a natural IgG $\mathrm{mAb}$, which exists as a tetramer made up of two light chain-heavy chain pairs. The researchers reasoned that a simple approach would be to take one light chainheavy chain pair from an existing IgG $\mathrm{mAb}$ and join it to a light-heavy pair from a different $\mathrm{mAb}$.

The problem was the researchers needed to find a way to efficiently generate only the desired hybrid antibody. Normally when multiple antibody chains are expressed together without modification, they can pair in many different combinations that are difficult to separate biochemically. This can make large-scale manufacturing and purification impractical.

Christian Klein, head of oncology programs at Roche's Glycart AG subsidiary, told SciBX that the first piece of the puzzle was solved by Genentech Inc. almost 15 years ago. "A major breakthrough came in 1997 when Genentech developed the Knob-into-Hole [KiH] technology, which allows the heterodimerization of two different heavy chains. However, the problem remained that you would still get the wrong light chain associations," he said. Genentech is now a unit of Roche.

$\mathrm{KiH}$ technology works by replacing specific amino acids at the heavy chain dimerization interface so that two distinct heavy chain fragments heterodimerize with each other instead of homodimerizing with themselves. However, the two light chain fragments would still be capable of pairing indiscriminately with either heavy chain, leading to unwanted antibody products.
Roche has now solved that problem with its CrossMab technology, and the company provided proof of concept by joining its anti-VEGF antibodyAvastin bevacizumab with LC06, an anti-angiopoietin 2 (ANG2; ANGPT2) antibody. ANG2 is an angiogenesis-promoting growth factor that is upregulated in some tumors.

The breakthrough came when Wolfgang Schaefer, a research leader in molecular biology at Roche, figured out how to ensure the light chain of LC06 paired only with the heavy chain of LC06. The key was modifying LC06's light and heavy chains by swapping domains required for their interaction, so that they could only pair with each other and not with the light and heavy chains from Avastin (see Figure 1, "The CrossMab approach").

"Maybe it was naïve, but I was very fresh in biology and had a theoretical chemistry background, so I saw the antibody not as a protein complex but as a series of building blocks. That led to this breakthrough that no one had thought of before," Schaefer told SciBX.

Avastin is marketed by Genentech in the U.S. and by Roche elsewhere to treat numerous cancers. LC06 has been developed with Affitech A/S, and its development status was not disclosed.

In a xenograft mouse model of colorectal cancer, the new ANG2VEGF CrossMab inhibited tumor growth better than either Avastin or LC06 alone. The CrossMab also inhibited VEGF-induced corneal angiogenesis better than either antibody alone.

Results were published in the Proceedings of the National Academy of Sciences. Schaefer was lead author of the study and Klein was senior author.

"We need more straightforward, simplified tools. Some techniques are doable but are so baroque that it is hard to apply them on a modular scale," said Sachdev Sidhu, associate investigator at the Ontario Institute for Cancer Research and professor of molecular genetics at the University of Toronto. "This is simple and clear-cut and should be doable with any antibody."

Sidhu's lab is developing techniques to identify antibodies that bind new targets. This includes methods to enable antibody selection directly on cells and engineer synthetic antibodies in which the antigen-binding sites are designed from scratch.

Companies contacted by SciBX that specialize in the production of new antibody therapeutics were impressed by the approach.

Ivan Horak, CSO and CMO at Symphogen A/S, noted, "The major advantage of the present bispecific format, relative to other formats of the same class, is the similarity to the classical IgG architecture, which should secure many of the favorable drug development attributes of monoclonal antibodies including high expression levels, standardized CMC [Chemistry, Manufacturing and Control] development, high solubility, long shelf life and slow pharmacokinetics."

Janine Schuurman, director of strategic research at Genmab A/S, liked the versatility of the approach. "A big advantage of this CrossMab approach is that the method leaves the antigen-binding regions of the parental antibodies intact, so the method provides a way to convert, after some engineering, any given pair of antibodies into an almost natural bispecific IgG antibody," she said. 
Figure 1. The CrossMab

approach. Researchers at Roche have developed a new method to construct bispecific antibodies. Standard IgG mAbs, such as Avastin bevacizumab and the anti-angiopoietin 2 (ANG2; ANGPT2) antibody LC06 [a], consist of two identical light chain-heavy chain pairs, which each bind to the same antigen.

To construct a bispecific IgG-like CrossMab antibody that could bind to two antigens by

using two distinct light chain-heavy chain pairs, a two-step modification process was applied.

First, a dimerization interface was engineered into the C-terminus of each heavy chain using previously developed Knob-into-hole (KiH) technology [b]. This ensured that only a heterodimer of two distinct heavy chains, one from Avastin and one from LC06, would be efficiently formed.

Next, the constant heavy $1(\mathrm{CH} 1)$ and constant light $(\mathrm{CL})$ domains of LC06 were exchanged [c], keeping the variable heavy $(\mathrm{VH})$ and variable light $(\mathrm{VL})$ domains consistent. The exchange of the $\mathrm{CH} 1$ and $\mathrm{CL}$ domains ensured that the modified LC06 light chain would only efficiently dimerize with the modified LCO6 heavy chain, while the unmodified Avastin light chain would only efficiently dimerize with the Avastin heavy chain. Thus only the desired bispecific CrossMab would be efficiently formed.

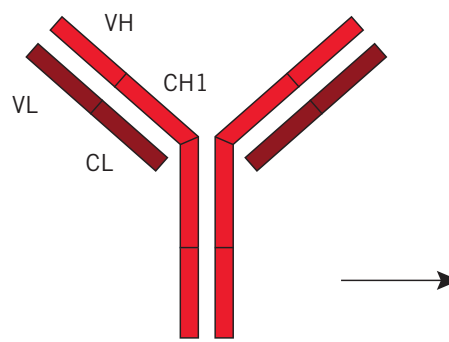

Anti-ANG2 LC06

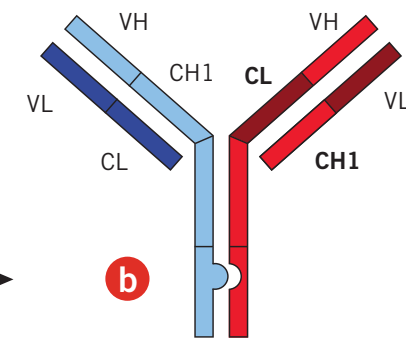

Anti-VEGF/ANG2 bispecific antibody
Genmab's DuoBody bispecific platform is based on Fab-arm exchange, which also generates bispecific antibodies with a natural IgG structure. ${ }^{3}$ Schuurman told SciBX that the DuoBody platform can yield regular and fully functional IgG-like bispecific molecules with limited mutations, but additional details about the technology have not been disclosed.

The first therapeutic DuoBody candidate against undisclosed targets is in preclinical development, with an IND candidate expected in 2015.

\section{Scaling up}

Joerg Regula, head of protein analytics at Roche Penzberg, said the company has tested the production of this antibody using a largescale, conventional Chinese hamster ovary $(\mathrm{CHO})$ cell expression system and has demonstrated yields and quality comparable to those of conventional antibody production.

"The plan is to bring several bispecific antibodies based on the CrossMab technology to clinical development over the next years," Klein said.

The key will be to find targets appropriate for the approach. Unlike normal IgG antibodies, which contain two antigen-binding regions that act on the same single target, CrossMabs only have one antigenbinding domain per target. In some cases, both antigen-binding arms are needed to increase the overall binding strength, or avidity, to a particular target.

"Loss of homodimerization is obviously a potential drawback of the

a

"The plan is to bring several bispecific antibodies based on the CrossMab technology to clinical development over

-Christian Klein, Roche present approach, as there may be situations where bivalent binding is required to bring about effective clearance of soluble molecules or cross-linking of cellular targets. This could limit the therapeutic efficacy of the approach, if any of these mechanisms are essential," said Symphogen's Horak.

Sidhu noted, "Really, it's good to have both bivalent, bispecific and monovalent, bispecific antibodies, and the only way to know what will work best is to try them in a particular system."

Indeed, last December at the American Society for Hematology meeting, Klein's team described a different attempt to develop a bispecific, tetravalent anti-ANG2-VEGF antibody. The antibody was generated by fusing two single-chain variable antibody fragments (scFv) from LC06 to the C-terminus of Avastin.

Klein told SciBX the antibody had no advantage in efficacy in preclinical studies over the CrossMab. He noted that tetravalent antibodies also could increase the risk of immune complex formation, so the CrossMab technique might be safer. Tetravalent antibodies can potentially bind to four antigens, increasing the possibility of creating large antigen-antibody complexes.

Horak and Sidhu both agreed that the immunogenicity of CrossMabs could not be ruled out, with Horak suggesting that Phase I studies were needed.

Symphogen is taking a different approach to hitting multiple antigen targets. The company is developing therapeutics that are mixtures of different mAbs. Horak said this avoids target restrictions based on 


\section{ANALYSIS}

physical proximity or steric hindrance and allows the targeting of multiple antigens at different doses, avoiding dose-limiting toxicities.

Roche has filed patents covering the CrossMab approach. The $\mathrm{KiH}$ technology used in the approach was patented by Genentech, but Genentech researchers were not involved in the new paper.

Cain, C. SciBX 4(28); doi:10.1038/scibx.2011.783

Published online July 21, 2011

REFERENCES

1. Schaefer, W. et al. Proc. Natl. Acad. Sci. USA;

published online June 20, 2011; doi:10.1073/pnas.1019002108

Contact: Christian Klein, Roche Glycart AG, Schlieren, Switzerland

e-mail: christian.klein.ck1@roche.com
Contact: Wolfgang Schaefer, Roche Diagnostics $\mathrm{GmbH}$, Penzberg, Germany

e-mail: wolfgang.schaefer@roche.com

2. Beck, A. et al. Nat. Rev. Immunol. 10, 345-352 (2010)

3. Van der Neut Kolfschoten, M. et al. Science 314, 1554-1557 (2007)

COMPANIES AND INSTITUTIONS MENTIONED

Affitech A/S (CSE:AFFI), Copenhagen, Denmark

Genentech Inc., South San Francisco, Calif.

Genmab A/S (CSE:GEN), Copenhagen, Denmark

Ontario Institute for Cancer Research, Toronto, Ontario, Canada

Roche (SIX:ROG; OTCQX:RHHBY), Basel, Switzerland

Symphogen A/S, Copenhagen, Denmark

University of Toronto, Toronto, Ontario, Canada 